Aktuelles vom SABCS zum fortgeschrittenen Mammakarzinom:

\section{Novartis präsentiert Subgruppenanalysen zu Afinitor ${ }^{\circledR}$ (Everolimus)}

Afinitor $^{\circledR}$ (Everolimus, RAD001) ist seit Juli 2012 für die Behandlung des Hormonrezeptorpositiven, HER2/neu-negativen, fortgeschrittenen Mammakarzinoms zugelassen. Die Grundlage der Zulassung bildet die Phase-III-Studie BOLERO-2. Im Rahmen des diesjährigen San Antonio Breast Cancer Symposium (SABCS) wurde erstmals die zentrale Auswertung der vordefinierten Subgruppen vorgestellt.

Signifikante Verlängerung des medianen PFS in allen Subgruppen

Alle Patientinnen profitieren signifikant bezüglich des medianen progressionsfreien Überlebens (PFS) von der Therapie mit Everolimus plus Exemestan (HR = 0,45; 95\%-KI: 0,38-0,54; $\mathrm{p}<0,0001)$ - unabhängig von Alter, Anzahl der Vortherapien sowie Ort der Metastasierung. Sowohl Patientinnen mit viszeralen Metastasen (medianes PFS 8,31 Monate unter Everolimus plus Exemestan vs. 2,89 Monate bei Placebo plus Exemestan; HR $=0,46$ ) als auch Patientinnen ohne viszerale Beteiligung (medianes PFS 16,59 vs. 5,82 Monate bei Placebo plus Exemessamtüberleben steht noch aus [1]. Mammakarzinom? $\tan ; \mathrm{HR}=0,27$ ) zeigten einen signifikanten Nutzen von der Kombination Everolimus plus Exemestan. Auch die Erstlinienpatientinnen in der fortgeschrittenen Situation erzielten einen signifikanten Vorteil bezüglich des medianen PFS. Dieses Kollektiv erreichte ein medianes PFS von 15,01 Monaten in der Everolimus-plus-Exemestan-Gruppe versus. 6,80 Monaten in der Placeboplus-Exemestan-Gruppe $(\mathrm{HR}=0,38)$ [1].

In den Interimsanalysen der BOLERO-2-Studie zeigte sich dabei ein positiver Trend zum längeren Gesamtüberleben für Everolimus und Exemestan, die finale Auswertung zum Ge-

Paradigmenwechsel beim fortgeschrittenen

Die zentrale Auswertung der BOLERO-2-Studie zeigte bereits ein medianes PFS von 11,0 Monaten unter der Kombination von Everolimus und Exemestan versus 4,1 Monaten unter Placebo und Exemestan (HR = 0,38; 95\%-KI: 0,310,$48 ; \mathrm{p}<0,0001$ ) [1] (Abb. 1). Die häufigsten Nebenwirkungen über alle Grade (Inzidenz $\geq 30 \%$ ) waren Stomatitis,

Unabhängige Auswertung
HR=0,38 (95\%-Kl: $0,31-0,48)$
Log-rank p-Wert: <0,0001
Kaplan-Meier-Kurven
EVE 10 mg + EXE: 11,0 Monate
PBO + EXE:
Hautausschlag, Müdigkeit, Diarrhö und vermin-

Abb. 1. $18 \mathrm{Mo}-$ nate Follow-up BOLERO-2: Everolimus mit medianem PFS von

11 Monaten; nahezu 1 Jahr ohne Progression, mod. nach [1].

$\mathrm{EVE}=$ Everolimus $\mathrm{EXE}=$ Exemestan $\mathrm{PBO}=$ Placebo derter Appetit [1]. Die häufigsten Nebenwirkungen vom Grad 3/4 (Inzidenz $\geq 2 \%$ ) waren Stomatitis, Hyperglykämie, Fatigue, nicht-infektiöse Pneumonitis und Diarrhö [2].

BOLERO-2 ist eine zulassungsrelevante randomisierte, doppelblinde, Placebo-kontrollierte, multizentrische Phase-III-Studie. In dieser Studie wurden 724 postmenopausale Frauen mit Hormonrezeptor-positivem, HER2/neu-negativem, fortgeschrittenem Brustkrebs und einem Durchschnittsalter von 62 Jahren untersucht, die ein Rezidiv oder eine Progression nach einer Therapie mit Letrozol oder Anastrozol vorwiesen.

Der mTOR-Inhibitor wird in Kombination mit Exemestan bei postmenopausalen Frauen zur Therapie des Hormonrezeptor-positiven, HER2/ neu-negativen fortgeschrittenen Mammakarzinoms ohne symptomatische viszerale Metastasierung angewendet, nachdem es zu einem Rezidiv oder einer Progression unter einem nichtsteroidalen Aromataseinhibitor gekommen ist [3]. Die Arbeitsgemeinschaft Gynäkologische Onkologie (AGO) hat die Kombinationstherapie bereits als Empfehlung in ihre Leitlinien für 2012 aufgenommen [4].

\section{Literatur}

1 Piccart M, et al.: Final progression-free survival analysis of BOLERO-2: a phase III trial of everolimus for postmenopausal women with advanced breast cancer. SABCS 2012, poster P6-04-02.

2 Piccart M, et al.: Everolimus for postmenopausal women with advanced breast cancer: updated results of the BOLERO-2 phase III trial. J Clin Oncol 2012;30(suppl) abstr \#559.

3 Fachinformation Afinitor ${ }^{\circledR}$, Stand: Juli 2012. www.fachinfo.de/pdf/01/19/011942.pdf

4 Arbeitsgemeinschaft Gynäkologische Onkologie e.V. (AGO): Guidelines Breast Version 2012.1D. www.ago-online.de/de/fuer-mediziner/leitlinien/mamma

Weitere Informationen bei

Novartis Pharma GmbH

Yasmin Messer

Roonstraße 25, 90429 Nürnberg

yasmin.messer@novartis.com

\title{
PharmaTicker+++ PharmaTicker+++ PharmaTicker+++ PharmaTicker+++
}

Fresenius Biotech. Der trifunktionale Antikörper Catumaxomab $\left(\right.$ Removab $\left.^{\circledR}\right)$ ist zugelassen für die Behandlung des malignen Aszites bei Patienten mit EpCAM-positiven Tumoren. Die Ergebnisse der neuen Phase-IIIb-Studie CASIMAS bestätigen die Daten der Zulassungsstudie. Die Therapie mit Catumaxomab erspart Patienten belastende Parazentesen und lindert nachhaltig Aszitesbedingte Symptome. Dies führt zu einer deutlich stabileren Lebensqualität, einem wichtigen Therapieziel bei fortgeschrittenen Krebserkrankungen. adam $\mathrm{GmbH}$ - Health Public Relations

Colleen Barbara Adam

c.adam@adamhealth.de
AGO Breast / German Breast Group. Das lösungsmittelfreie nab-Paclitaxel (Abraxane ${ }^{\circledR}$ ) hat sich bereits beim metastasierten Mammakarzinom wegen seiner überlegenen Wirksamkeit und Verträglichkeit im Vergleich zu konventionellen Taxanen bewährt. Jetzt untersuchen die AGO Breast und die German Breast Group in den Studien GeparSepto und GAIN-2, inwieweit der Einsatz von nab-Paclitaxel die Effektivität der neoadjuvanten und adjuvanten Therapie beim frühen Brustkrebs weiter verbessern kann.

Selinka Schmitz PR GmbH

Nicole Zeuner

nicole.zeuner@selinka-schmitz-pr.de
AstraZeneca. Auf dem diesjährigen 35. CTRCAACR Brustkrebs-Symposium in San Antonio wurden neueste Daten zum Gesamtüberleben von Frauen mit Hormonrezeptor-positivem, lokal fortgeschrittenem oder metastasiertem Mammakarzinom vorgestellt, die eine Therapie mit 500 mg Faslodex ${ }^{\circledR}$ (Fulvestrant) erhalten hatten. Die Studiendaten zeigen eine Verbesserung des Gesamtüberlebens von median 4,1 Monaten bei Frauen, die mit Faslodex ${ }^{\circledR} 500$ mg vs. Faslodex ${ }^{\circledR}$ $250 \mathrm{mg}$ behandelt wurden.

AstraZeneca GmbH

Florian Dieckmann

florian.dieckmann@astrazeneca.com 